Article

\title{
Interfacial Characterization by Pull-Out Test of Bamboo Fibers Embedded in Poly(Lactic Acid)
}

\author{
Quentin Viel $^{1,4}$, Antonella Esposito ${ }^{1, *}$ (D) , Jean-Marc Saiter ${ }^{2}$, Carlo Santulli ${ }^{3, *}$ \\ and Joseph A. Turner ${ }^{4}$ \\ 1 Normandie Univ, UNIROUEN Normandie, INSA Rouen, CNRS, Groupe de Physique des Matériaux, \\ 76100 Rouen, France; quentin.viel@etu.univ-rouen.fr (Q.V.); antonella.esposito@univ-rouen.fr (A.E.) \\ 2 AMME-LECAP EA 4528 International Laboratory, Institute for Materials Research, \\ Université de Rouen BP 12, 76801 Saint-Etienne du Rouvray CEDEX, France; jean-marc.saiter@univ-rouen.fr \\ 3 School of Architecture and Design (SAAD), Università degli Studi di Camerino, Viale della Rimembranza, \\ 63100 Ascoli Piceno, Italy \\ 4 AMME-ATEAM International Laboratory, Department of Mechanical and Materials Engineering, \\ University of Nebraska-Lincoln, Lincoln, NE 68588-0526, USA; jaturner@unl.edu \\ * Correspondence: antonella.esposito@univ-rouen.fr (A.E.); carlo.santulli@unicam.it (C.S.); \\ Tel.: +33-2-3295-5083 (A.E.); +39-334-1737527 (C.S.)
}

Received: 25 December 2017; Accepted: 16 January 2018; Published: 19 January 2018

\begin{abstract}
In this work, the apparent shear strength at the interface between a bamboo fiber and the surrounding poly(lactic acid) (PLA) matrix is quantified. A method for processing pull-out test samples within a controlled embedded length is proposed and the details of the test procedure are presented, along with a critical discussion of the results. Two series of samples are considered: untreated and mercerized bamboo fibers from the same batch, embedded in the same polyester matrix. Electron and optical microscopy are used to observe the fiber-matrix interface before and after the test, and to identify the failure mode of each sample, especially as regards the occurrence of fibrillation in the fiber bundles. The values of apparent interfacial shear strength are calculated only for regular fibers successfully pulled out from the matrix, and reported with their statistical variations. Mercerization, whose efficiency was proven by Fourier transform infrared (FTIR) spectroscopy, did not appear though to improve the quality of the interface $\left(\tau_{\text {app }}=7.0 \pm 3.1 \mathrm{MPa}\right.$ for untreated fibers and $\tau_{\text {app }}=5.3 \pm 2.4 \mathrm{MPa}$ for treated fibers).
\end{abstract}

Keywords: bamboo biomass; biodegradable composites; pull-out; surface treatments; mercerization

\section{Introduction}

Processing 100\% green composites implies that biosourced (biodegradable) polymeric resins can be conveniently reinforced by natural materials, such as plant-based fibers, for example jute, hemp, flax and bamboo fibers [1-5]. Bamboos are one of the most abundant ligno-cellulosic plants produced in the world [6]. There are so many different species of bamboos, and some of them grow so fast (up to $120 \mathrm{~cm}$ per day), that no other source of vegetable fibers could be considered more renewable. On the other hand, it is not obvious how to control from a botanical point of view the supply of bamboo from tropical forests, due to their degradation, especially when classified as "ambiguous lands", i.e., in principle state-owned yet used by low income people for harvesting, e.g., bamboo shoots [7]. For this reason, a possibility which has been explored is for example the extraction of lignin from steam exploded bamboo for the production of epoxy resins [8].

A more valuable yet sustainable perspective is nonetheless the possibility to produce composites by using bamboo fibers as the reinforcement for biodegradable matrices [9]. In this regard, the use of bamboo fibers, also due to the variable quality of supply, cannot always be recommended, because 
the difficult predictability of their properties has an effect also on those of composites produced with them. For example, due to the variable presence of defects in the fibers, introducing longer fibers in the load direction does not always result in substantial and repeatable improvement of composite performance, as, e.g., reported in a study on bamboo fiber/starch-based composites [10]. A further issue, which heavily contributes to the variability of bamboo fibers properties, is that, as it is the case for all ligno-cellulosic fibers, their morphology can be very variable in terms of diameter, hollow size and geometry, elliptical shape of the section, etc. [11-13].

Polymers synthesized from naturally sourced monomers, such as poly(lactic acid) (PLA), have in contrast quite controllable properties and are therefore very suitable for the production of fully sustainable composites with vegetable fibers: a significant amount of literature does exist on these materials [14]. A major aspect to be controlled is therefore the quality of the fiber-matrix interface, that can be measured on fiber composites by a number of possible methods, among which is pull-out testing [15].

An early study on flax fiber pull-out from polyethylene matrix proposed that the same methods used in the case of traditional fibers, such as glass or carbon fibers, could work as well, despite the peculiar structural characteristics of lignocellulosic fibers [16]. Following this, pull-out testing proved suitable to offer sufficiently reliable values of the apparent interface shear strength on a number of different lignocellulosic fibers [17]. Pull-out tests of sisal fibers embedded at three-millimeter depth in polyester blocks of 10-mm side and then stretched to failure confirmed, despite a very large standard deviation, the effectiveness of mercerization in improving fiber-matrix interface strength [18].

As for bamboo fibers, the significant influence of environmental factors on the adhesion strength between fiber and matrix has already been highlighted [19]. However, in general, the measurement of interface strength has found so far only quite limited coverage in the literature, despite the fact that substantial issues are still perceived in the achievement of sufficient properties for PLA/bamboo fiber composites $[2,6,11,20]$. Recent work pointed out that information about practical adhesion of bamboo fibers with thermoplastic polymer matrices could be obtained from different parameters from pull-out tests, such as interfacial shear stress, radial tensile stress and theoretical work of adhesion: the study indicated a better interface for bamboo fibers with poly(vinylidene fluoride) (PVDF) rather than with polypropylene (PP), although much weaker than for glass fibers [21].

The measurement of apparent shear strength can be not completely accurate, since the pull-out process may be friction-dominated in bamboo fiber systems, and friction is not considered by this parameter [22]. Accurate measurements of interfacial adhesion in bamboo fibers are also cumbersome for other reasons, such as the variable adhesion of filaments to form bundles (which makes them easily prone to fibrillation) and the need to account also for cross-sectional complex shapes and variable sizes (which requires multiple measurements of the diameter across the same section). Notwithstanding these limitations, it is worth considering whether the application of a simple method for measuring the apparent interfacial shear strength with values of diameter obtained under reasonable assumptions would supply any suitable indication for the improvement of composite properties. This approach may be simplistic in comparison with other approaches, such as local analysis and computational predictions [23], well adapted when both the matrix and the fibers have quite regular and predictable properties. However, when it comes to natural materials, whose supply is not subjected to a rigorous quality control from the producer as for synthetic ones, but only possibly to some selection of the most suitable samples, predictability is a real issue: for this reason, experimental approaches, which give access to the average properties of the entire population under sensible assumptions, can provide reasonably good estimates of the properties. In spite of this, the positive influence of maleinisation and silane treatment on polypropylene/bamboo composites interfacial shear strength was highlighted [24].

In this work, measurements of the apparent interfacial shear strength were carried out by performing pull-out tests on bamboo technical fibers embedded in thin PLA matrices. Fibers belonging to the same batch were used either as received or after mercerization, embedded in the same polymeric 
matrix. A procedure was used to obtain samples with an improved control over the embedded length, and a critical approach to the experimental results was used to select and interpret them.

\section{Materials and Methods}

\subsection{Materials}

A commercial grade of poly(lactic acid) (PLA) denoted PLI 003, with density $\rho=1.25 \pm 0.05 \mathrm{~g} / \mathrm{cm}^{3}$ and melting temperature around $145-155^{\circ} \mathrm{C}$, was purchased from NaturePlast (Caen, France). Bamboo Fibers Technology (Lahontan, France) imports bamboo fibers and fillers from Thailand, and proposes different grades (G1 to G6, from short fibers to extremely fine particles). Only short fibers belonging to grades G1 and G2 were considered in this work (fibers $5-150 \mathrm{~mm}$ long). Their tapped density is $90 \mathrm{~kg} / \mathrm{m}^{3}$ and their humidity is comprised between $8 \%$ and $15 \%$ in normal ambient conditions. The batch of fibers used in this work was extracted from plants grown in the wild forests of northern Thailand, Province of Lamphun. Several genera and species of bamboos are recorded in Thailand [25], but the major type of bamboos found throughout the country is tropical bamboo, also referred to as sympodial bamboo [26]. Unfortunately, there has been no systematic survey of the vegetation in Thailand so far, so that in particular, no assessment of the bamboo resources from Thailand has been made [27]. Thailand lacks a proper vegetational system because the myriad of nomenclature have never been sorted and even ecologists are often unable to get reliable names for their studies [28]. In Thailand, wild bamboos can be found in three of the four subtypes of forest, i.e., dry evergreen forests (DEF), mixed deciduous forests (MDF) and dry dipterocarp Forests (DDF). The fibers used in this work were extracted from plants grown in MDF where, according to Prachayo [29], only one species is found (Dendrocalamus strictus). It is worth mentioning, however, that Thailand's MDF are a form of degraded forest comprising a mixture of evergreen and deciduous trees organized in three layers, with the middle layer typically dominated by bamboos, and that it has been recently found that all bamboos in MDF are sympodial, but not necessarily belonging to the same species [30]; one may occasionally encounter clustered Gigantochloa albociliata, Bambusa tulda and Cephalostachyum pergracile [31]. The fibers were mechanically extracted from the bamboo stem by hatching, and then their size was gradually reduced from G1 to G6 by mechanical grinding. Several fibers belonging to the same batch were used either as received or after mercerization. Mercerization was performed with sodium hydroxide ( $\mathrm{NaOH})$ (Sigma-Aldrich, St. Louis, MI, USA, ACS reagent $\geq 97.0 \%$ ).

\subsection{Methods}

\subsubsection{Preparation of the Samples}

A preliminary naked-eye inspection allowed separating regularly-sized from clearly distorted and/or defective fibers. A typical cross-section of bamboo fiber bundles inside the culm presents an irregular form (bean shape) with a size variation depending on their position across the bamboo wall. Once extracted, each technical fiber, irrespective of its initial position, contains many elementary fibers (whose cross-section is either pentagonal or hexagonal) arranged in honeycomb patterns [32]. Whatever the process of extraction, natural fibers have variable cross-sectional shape, even throughout the same batch. Indeed, when the fibers are extracted, they are partially broken resulting in different cross-sectional shapes and sizes [12]. Optical microscopy revealed that the fibers selected from the batch used in this work were statistically dispersed, both in shape and size, with a cross-sectional shape mostly intermediate between circular and elliptical (Figure 1). Initially, the apparent diameters of the technical fibers used in this work (estimated from a side view by optical microscopy) were found to range from $200 \mu \mathrm{m}$ to $1 \mathrm{~mm}$. Some of the fibers out of the batch were also mercerized; mercerization is likely to help loosening the bundles and separating them into elementary filaments without inducing any significant modification of their structural components (crystallinity index and amount of native cellulose) [33]. For mercerization, the fibers were immersed in a water solution containing $5 \mathrm{wt} \%$ sodium 
hydroxide $(\mathrm{NaOH})$ for $24 \mathrm{~h}$ at room temperature: the long period of exposure to sodium hydroxide at a not very high concentration is expected to allow obtaining fibers with modified surfaces but equivalent structural properties (this will be dealt with in Section 3). After the treatment, the fibers were recovered by a plastic tray with filter paper and washed with filtered water in a Büchner flask. Finally, the fibers were placed on a plate coated with an aluminum foil and oven dried at $80^{\circ} \mathrm{C}$ for $24 \mathrm{~h}$.
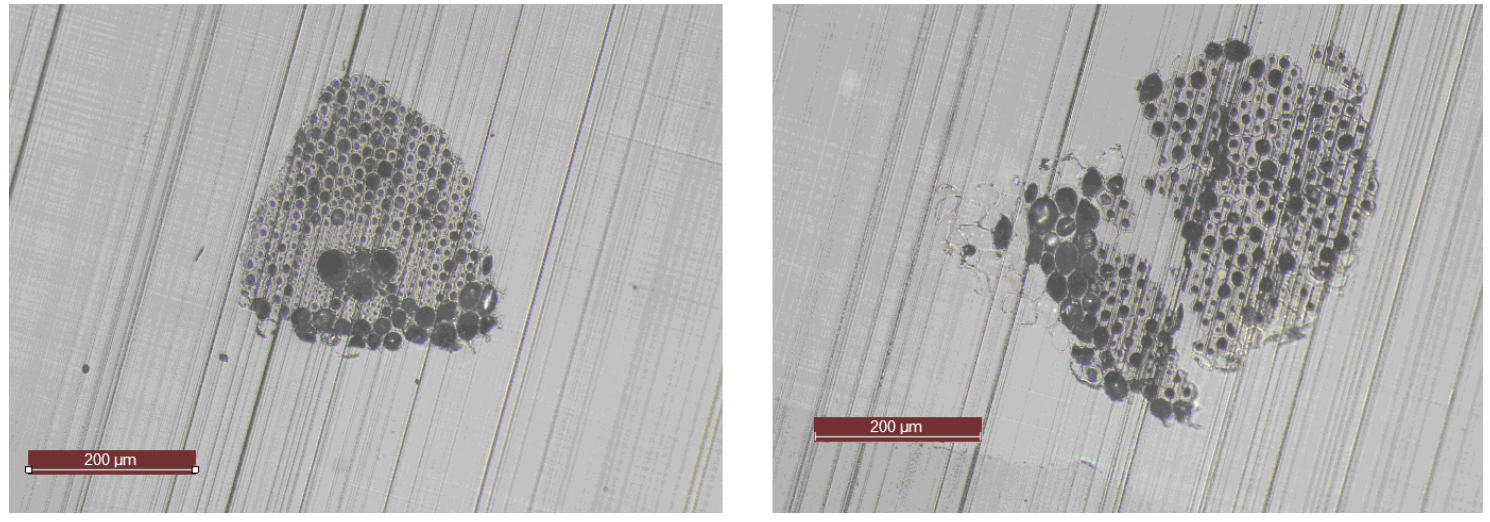

Figure 1. Cross-section of two bamboo fibers randomly extracted from the batch used in the study and imaged by optical microscopy.

Thin PLA disks were created by melting a given amount of dried pellets and then compressing them with a hydraulic manual press (Specac 25T, Orpington, UK). The thickness of the PLA disks was carefully controlled, as it represents a key parameter for pull-out tests (length of fiber embedded in the polymer matrix). The control over the thickness was ensured by (1) using round washers with known thicknesses $(h)$ and internal diameters $\left(D_{i}\right)$, and (2) determining the mass of PLA required to fill the volume inside each washer (Equation (1)).

$$
m=\pi\left(\frac{D_{i}}{2}\right)^{2} h \rho
$$

The procedure to obtain the PLA disks is the following: (1) two Teflon films and two aluminum foils are cut to the internal diameter of the washer; (2) both the Teflon films and the first aluminum foil are used in their pristine form, whereas the second aluminum foil is hole-punched as many times as the number of samples to be prepared; (3) a sandwich is obtained by stacking (in order) one Teflon film, the first aluminum foil, the washer filled with the PLA pellets, the second aluminum foil and another Teflon film (Figure 2a). The first aluminum foil acts as a support for the polymer sample along with the second aluminum foil, which is hole-punched to expose the PLA matrix in the areas where the bamboo fibers will be inserted. The sandwich is inserted between the hot plates of the hydraulic press (temperature controller set to $200{ }^{\circ} \mathrm{C}$, with the polymer not exceeding $170-175{ }^{\circ} \mathrm{C}$ ) and a force of 2 tons (10 to $30 \mathrm{MPa}$ depending on the external diameter of the washer) is applied for 2 min. After cooling down to room temperature, the thickness of the disks is checked using a caliper. Four washers with different thicknesses $(0.84,0.15,0.65$ and $0.55 \mathrm{~mm})$ and internal diameters (34.0, $38.2,44.6$ and 44.0 respectively) were used. The PLA disks were cut to produce as many samples as the number of holes punched in the second aluminum foil. In the meantime, the fibers were held in the oven at $80^{\circ} \mathrm{C}$ to avoid any water uptake, as the presence of water is known to weaken their interface with polymers [20]. The fibers were embedded, one by one, in every PLA precut disk heated between the hot plates of the press. Attention was paid to keep them as perpendicular as possible with respect to their surface and to force them to the bottom of the layer. 


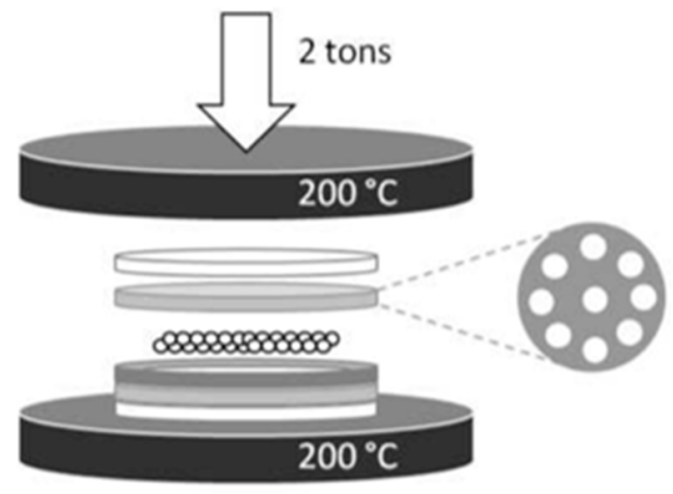

(a)

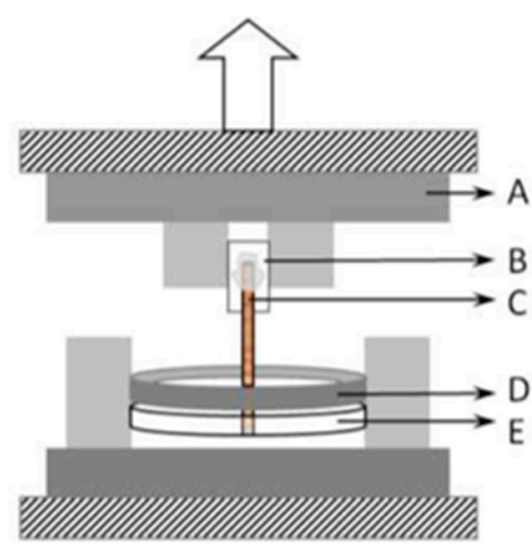

(b)

Figure 2. Procedure to obtain and test pull-out-samples. (a) creating poly(lactic acid) (PLA) thin disks. Between the hot plates of the hydraulic press (stacked from the bottom to the top): a Teflon film, an aluminum foil, a washer filled with PLA pellets, a hole-punched aluminum foil and another Teflon film. (b) placing samples on the Bose device: (A) clamp, (B) cardboard, (C) free tip of the bamboo fiber, (D) washer, and (E) PLA thin disk.

\subsubsection{Characterization of the Fibers and Pull-Out Tests}

The bamboo fibers were characterized by Fourier transform infrared (FTIR) spectroscopy before the alkali treatment, right after the alkali treatment (without washing) and after washing in filtered water. Prior to measurement, the fibers were dried and compacted into pellets ( $16 \mathrm{~mm} \varnothing)$ by the hydraulic manual press (Specac 25T). Several spectra were recorded on different areas of each pellet by a Varian 4100 FTIR device (Excalibur Series, Agilent, Santa Clara, CA, USA) in attenuated total reflection (ATR) mode with a mono-reflection diamond crystal (Pike MIRacle). Qualitative comparisons were made to evaluate the consequences of the alkali treatment on the chemical composition of the fibers. As the cross-sectional dimensions of a given bamboo fiber can vary along its length, each fiber candidate for pull-out testing was placed between two glass slides and observed by optical microscopy (Olympus BX51/BX52). Several measurements (at least 5) were performed to determine the variation of the apparent diameter along the length of interest at distances of around $200 \mu \mathrm{m}$ from each other. The average of the apparent diameters measured for each bamboo fiber was considered as its equivalent diameter, i.e., the diameter of a virtual fiber having a circular cross-section and the same mechanical behavior (in terms of pull-out response).

The pull-out tests were performed using a Bose ElectroForce 3200 device (TA Instruments, New Castle, DE, USA) with titanium T/C grips (maximum applied force $22.5 \mathrm{~N}$ ). The locking system was ensured by hex drive nuts, which facilitated the placement of the samples in the clamps. For each sample, the free tip of the fiber was fixed with MultiTemp ${ }^{\circledR}$ glue on a cardboard tab, which was clamped more easily than the fiber itself. As for the PLA thin disk, a washer was placed between the clamps to prevent sliding. The load vs. displacement curves were recorded by the software WinTest (TA Instruments, New Castle, DE, USA). All the tests were performed with a loading rate of $0.005 \mathrm{~mm} / \mathrm{s}$. The sample placement on the Bose device is sketched in Figure $2 \mathrm{~b}$.

A preliminary evaluation of the possibility to perform successful pull-out tests led to the observation of two ineffective outcomes of the test: either the fibers broke before the interface failed or, less frequently, the fiber tip and/or the matrix thin disk slid between the grips. The experimental results were classed as regular or non-regular depending not only on the global shape of the curve recorded during the pull-out test, but also on the aspect of the fiber tip after extraction from the matrix and the aspect of the hole left behind. Systematic observations of the fiber tip and the holes by scanning electron microscopy (SEM) were carried out after each pull-out test to decide whether the test was 
successful or not, therefore helping with selection and interpretation of the curves. The inspection of the fractured samples was carried out using an FEI Quanta 200 FEG high resolution field emission scanning electron microscope (FEI Company, Hillsboro, OR, USA). The results classed as regular, where no obvious fibrillation occurred, were used to estimate the apparent interfacial shear strength, as described and discussed in Section 3.

\section{Results and Discussion}

Pull-out tests consist of embedding a fiber in a matrix and then performing a tensile test by gripping the free tip of the fiber (top) and the matrix (bottom) to pull them apart (Figure 2b). The result typically expected for this kind of test is the fiber being pulled out of the matrix without breaking, meaning that adhesive failure (debonding at the fiber/polymer interface) rather than cohesive failure (either in the fiber or in the matrix) occurs. The force vs. displacement curves are used to determine the shear strength at the interface between the fiber and the matrix using the Kelly-Tyson equation, as suggested by Greszczuk (Equation (2)) [34] that can be written as:

$$
\tau_{\text {app }}=\frac{F_{\text {deb }}}{\pi D_{f} L_{e m b}}
$$

where $\tau_{a p p}(\mathrm{MPa})$ is the apparent interfacial shear strength, $F_{\text {deb }}(\mathrm{N})$ is the force required to debond the fiber from the matrix, $\pi D_{f}(\mathrm{~mm})$ is the perimeter of the fiber as $D_{f}(\mathrm{~mm})$ is the diameter, and $L_{e m b}(\mathrm{~mm})$ is the length of fiber embedded into the matrix.

Equation (2) provides a value of interfacial shear strength that is apparent because it depends on both the embedded length and the diameter of the fiber, which are usually assumed constant. A number of issues can be seen in the application of Equation (2) to pull-out tests on vegetable fibers. Major ones would concern the fact that the section of vegetable fibers is typically non-circular, presenting at least some ellipticity if not a more complex shape, which may be not accounted for by the method of diameter measurement. In practice, considering the diameter at five different locations along the length of the fiber, as described in Section 2.2.2, led to measuring values with a standard deviation between $0.7 \%$ and $7.5 \%$ and an average standard deviation of $3.1 \%$. As a whole, the main concentration of diameter values measured in this work reflected those reported by Rao and Rao [35], who found that most mechanically extracted bamboo fibers have a diameter between 200 and 350 microns.

The experimental approach suggested in this work gives a reliable value for $L_{e m b}$ and the debonding force $F_{d e b}$ is measured directly: the consequence of this fact is that the fiber perimeter $\pi D_{f}$ estimated with the assumption of a constant diameter is the parameter in Equation (2) with the highest uncertainty. Each fiber is unique and has a shape that varies over its entire embedded length; thus, estimates of fiber perimeter can be made only indirectly at best, unless the fiber is imaged while embedded in the matrix for example using X-ray microtomography ( $\mu$-CT) [36].

At least five possibilities exist for estimating the contact perimeter, and each one of these has its own advantages and disadvantages. For example, the perimeter of the hole left in the PLA matrix by the pulled-out bamboo fiber could be estimated by SEM, but this approach lacks precision and does not account for the fact that the perimeter is not uniform over the entire embedded length. Another approach would be to cut the fiber before embedding and taking an image of the cross-section, but cutting obviously introduces damage, which may reduce the fiber strength. Some authors assume a density and estimate the total fiber length to evaluate the perimeter by weighing a group of fibers [12], whereas others estimate the perimeter by the Wilhelmy method [34]. Finally, assumptions about the fiber cross-sectional shape can be made, such as the projection of a virtual cross-section whose diameter could be estimated from optical microscope images at several positions along the fiber length and that would behave equivalently to the real cross-section. In this work, the final choice was made to assume a circular equivalent fiber such that the perimeter is $\pi D_{f}$ with $D_{f}$ as the equivalent diameter, obtained as the average of five values of apparent diameter measured at different places along each fiber from a side view by optical microscopy. This approach is expected to give estimates for the perimeter that 
are larger than reality, in a way that the final estimates for the apparent interfacial shear strength are lower than reality (conservative estimate).

In Equation (2), $\tau_{\text {app }}$ was interpreted as if the fiber was loaded until debonding and then completely extracted from the matrix. $F_{d e b}$ was estimated as the maximum value of the experimental force vs. displacement curves, whereas $L_{e m b}$ was evaluated by measuring the thickness of the PLA thin disk. Figure 3 reports two examples of the typical load vs. displacement curves obtained in this work. In all cases the fiber was completely extracted from the polymer without clearly breaking. Figure 3a shows the ideal outcome for the pull-out tests performed in this work (smooth load vs. displacement curve until interface failure by fiber/polymer debonding). Results like these are the most appropriate to calculate $F_{d e b}$ and $\tau_{a p p}$ because none of the elementary fibers break during the test. On the other hand, the curve in Figure $3 \mathrm{~b}$ reveals that some intermediate failure occurred during the tensile part of the pull-out test. In fact, whenever the test is run on a technical fiber, the internal interfaces between the elementary fibers may debond at lower loads with respect to the outer interface, and break the bundle before debonding it from the matrix. Curves like this cannot be assuredly discarded, but can neither be used to determine $\tau_{\text {app }}$ according to Equation (2). In this case the stressed interface is not only the one connecting the outer surface of the technical fiber to the matrix, but also the one connecting the elementary fibers to each other within the same bundle. For this reason, even when the experimental curves seemed correct, the tip of each bamboo fiber extracted from the PLA matrix was observed by optical microscopy (example pictures on the top right corner of Figure $3 a, b$ ) to identify the failure mechanism associated with the pull-out test and to get a deeper insight in the nature of the constraints experienced by the fiber.
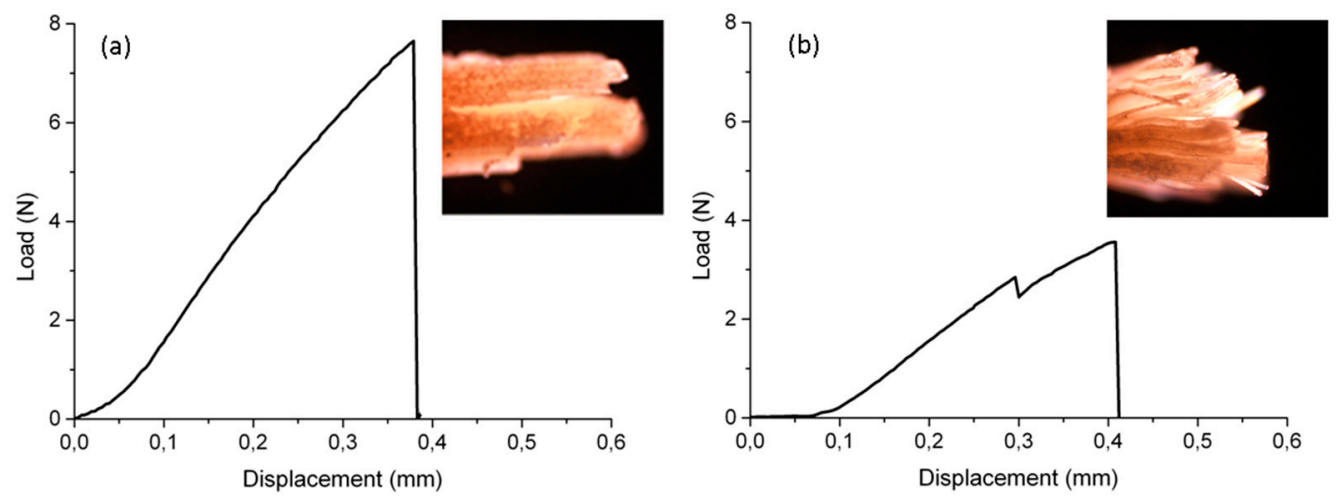

Figure 3. Typical load vs. displacement curves obtained by pull-out tests performed on bamboo fibers embedded in a PLA matrix: (a) adhesive failure at the bamboo/PLA interface and (b) adhesive failure combined with cohesive failure within the bamboo fiber. The pictures on the top right corner of each curve show the typical aspect of the fiber tips after the pull-out test: (a) regular and (b) non-regular fiber.

Regular fibers were defined as those that did not show an obvious fibrillation, i.e., separation of filaments, when being extracted from the polymer matrix (Figure 3a). Whenever fibrillation was clearly shown during fiber failure so that the tensile load could not be regarded as exclusively applied orthogonally to the fiber section, the fibers were defined as non-regular (Figure 3b). SEM images confirmed that successful pull-out tests were most frequently obtained from regular fibers, where the fiber tip remained aligned with the bulk of it during the whole loading event. Moreover, when the fiber behavior was non-regular, a load drop was frequently observed during loading, which can be attributed to the moment when the filaments started splitting from each other, leading to fibrillation. In general, fibrillation might be considered to improve fiber-matrix adhesion; as a matter of fact, mechanically induced fibrillation has been already used on bamboo-PLA composites to make them more suitable to processing techniques, such as extrusion, pelletizing and subsequent injection molding [37]. However, when fibrillation occurs, pull-out tests usually overestimate the value obtained for the interfacial shear 
strength because mechanical interlocking could contribute to a stronger connection of the technical fiber (through its elementary fibers) to the polymeric matrix.

Figure 4a shows the failure mode associated with non-regular pull-out-test curves; in this case, the technical fiber partially broke instead of getting pulled out of the matrix, meaning that the bamboo/PLA interface was not necessarily stressed to failure. In contrast, Figure $4 \mathrm{~b}$ shows the hole left in the matrix by a fiber pulled out during a regular pull-out test; here, only a few elementary fibers broke, proving that the interface actually stressed by the pulling action was essentially the one connecting the outer surface of the technical fiber to the polymer. It is worth noticing that the bamboo fiber shown in Figure $4 \mathrm{a}$ has an apparent diameter of about $200 \mu \mathrm{m}$, whereas the one shown in Figure $4 \mathrm{~b}$ is much thicker (about $500 \mu \mathrm{m}$ ). This is quite typical and indicates that, to avoid fibrillation, fibers should not only be regularly-sized and possibly defect-free, but also rather thick. In fact, a balance should be found between the cross-section resisting the uniaxial tensile stress (which depends exactly on the cross-sectional shape and size of the technical fiber) and the extent of the outer surface in connection with the matrix (which depends essentially on the equivalent diameter of the fiber and on the length of it actually embedded in the polymeric matrix, $\left.L_{e m b}\right)$. Considering only regular samples (12 values out of the 39 tests reported in this work), a value $\tau_{\text {app }}=7.0 \pm 3.1 \mathrm{MPa}$ was obtained.
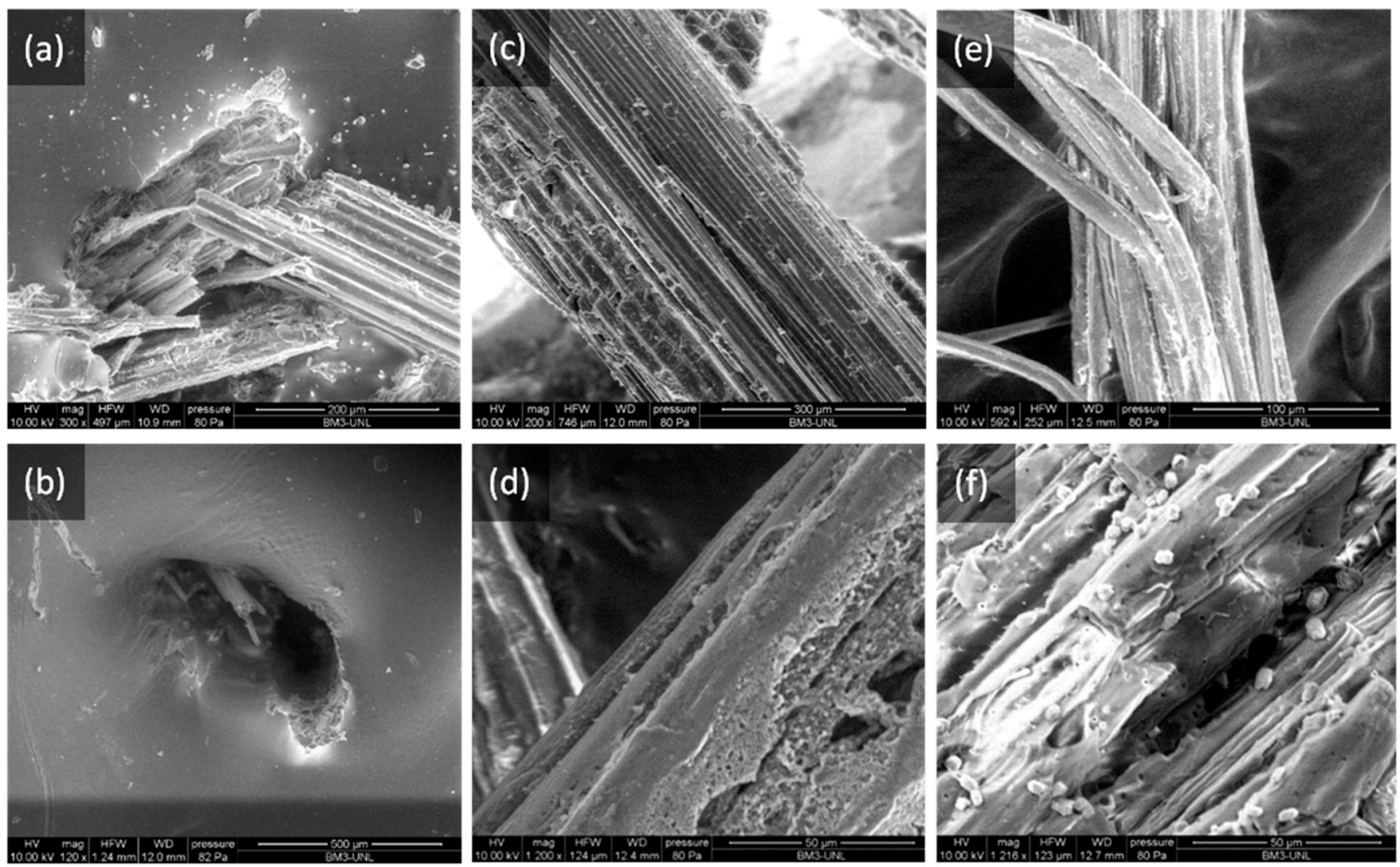

Figure 4. SEM images of (a) a technical bamboo fiber that broke (instead of debonding) during the pull-out test (apparent diameter of the fiber ca. $200 \mu \mathrm{m}$ ); (b) the hole left in the PLA matrix by a bamboo fiber successfully pulled-out during the test (diameter of the hole ca. $500 \mu \mathrm{m}$ ); (c) the surface of an untreated bamboo fiber (scale bar $300 \mu \mathrm{m}$ ); (d) the surface of a mercerized bamboo fiber (scale bar $50 \mu \mathrm{m}$ ); (e) the loosening of a technical bamboo fiber (bundle of elementary fibers) as a consequence of mercerization (scale bar $100 \mu \mathrm{m})$; (f) residual salts $(\mathrm{NaOH})$ left behind by mercerization in the absence of a proper washing procedure (scale bar $50 \mu \mathrm{m}$ ).

Tokoro et al. [20] used short bamboo fiber bundles (diameter $39.2 \mu \mathrm{m}$ and length $215 \mu \mathrm{m}$ before fabrication) to obtain PLA composites by injection molding. They performed micro-droplet tests to evaluate $\tau_{\text {app }}$ according to Equation (2) and found an average value of about 4.3 MPa (estimating that processing reduced both the diameter and the length to $21.3 \mu \mathrm{m}$ and $86.3 \mu \mathrm{m}$ respectively), but no statistical variations were given about these values. A clear comparison is difficult to make, because 
the samples used for this study have larger $D_{f}(200-480 \mu \mathrm{m})$ and $L_{e m b}(423-790 \mu \mathrm{m})$ and the results obtained for regular samples spread over a range going from 2.1 to $13.6 \mathrm{MPa}$. Fuentes et al. [38] performed fiber pull-out tests on technical bamboo fibers embedded in poly(vinylidene fluoride) (PVDF) and polypropylene (PP). The diameter of the fibers was not given, but their perimeter was estimated by the Wilhelmy equation applied to dynamic contact-angle measurements. They considered different $L_{e m b}(0.6$ to $5 \mathrm{~mm})$ and fit the experimental data with Greszczuk's model [35], finding a value of ultimate interfacial shear strength of about 7.5 MPa for PVDF (polar) and 3.4 MPa for PP (non polar). As a comparison, the value obtained here is quite close to the value obtained for PVDF, which is consistent considering that PLA is a fairly polar polymer. Le Duigou et al. [39] performed microbond tests on flax fibers and poly (L-lactic acid) (PLLA), and reported a value of interfacial shear strength of $15.3 \pm 3.3 \mathrm{MPa}$ calculated according to Equation (2) for fibers having an aspect ratio $\left(L_{e m b} / D_{f}\right)$ of $1.3 \pm 0.1$. Wong et al. [40] also used Equation (2) to evaluate the interfacial strength between flax fibers and PLLA. They obtained values in the range 8-17 MPa by pulling out fibers having $D_{f}$ in the range 100-160 $\mu \mathrm{m}$ and $L_{e m b}$ in the range 200-500 $\mu \mathrm{m}$. The comparison of all these values, especially when it comes to $D_{f}$, would be even more difficult if the ellipticity (or even higher geometrical complexity) of bamboo fiber sections were, as it should be, taken into account. In fact, this would require the measurement of the exact diameter of the fractured section, an example of which is reported in Figure 5. Such a procedure was not systematically pursued due to the difficulty of getting a section with obvious edges and as flat as possible, and may be the object of dedicated work in the future.

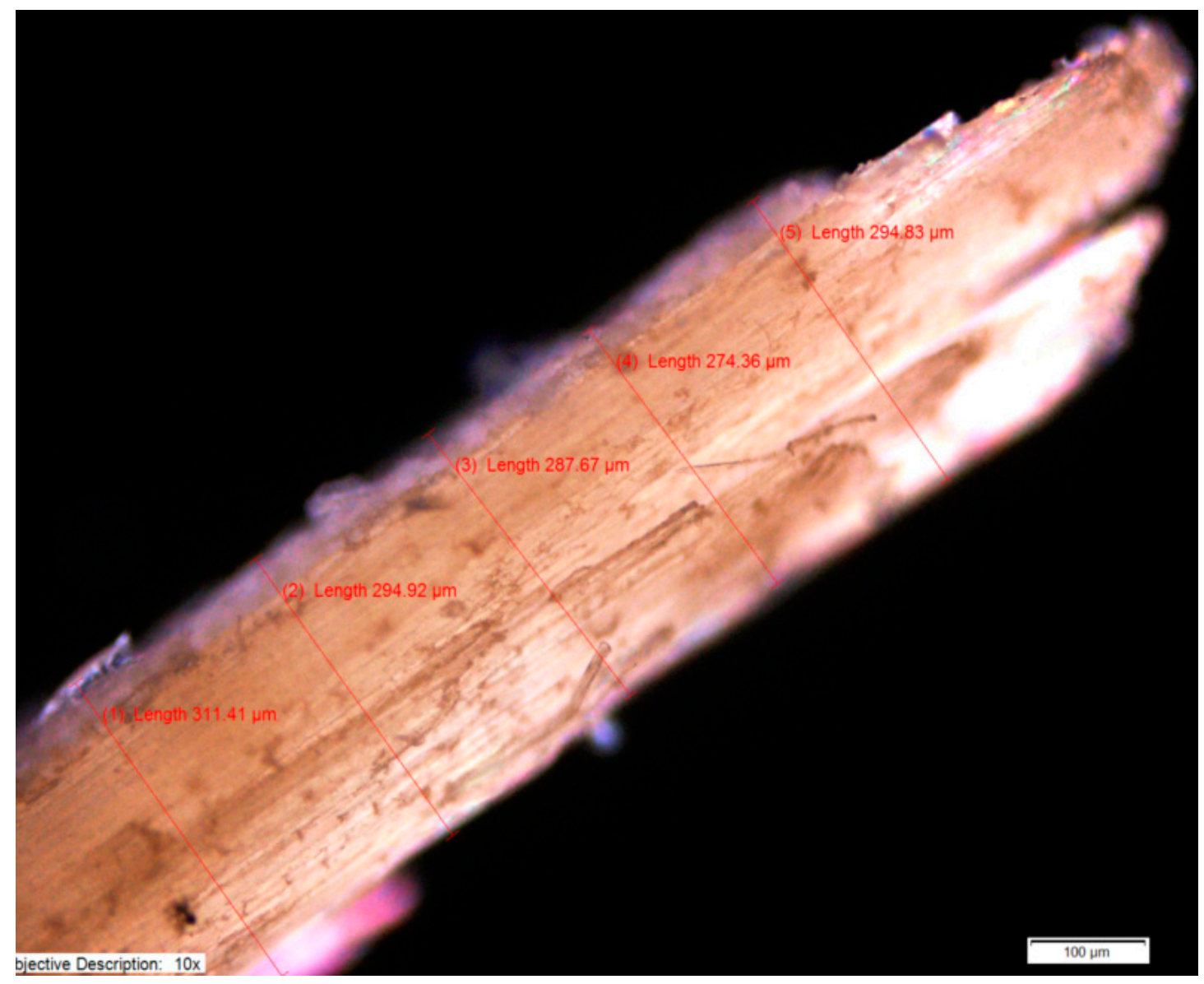

Figure 5. SEM image of fractured section of a bamboo fiber with tentative measurement of diameter.

The values of maximum load $\left(F_{\max }\right)$ recorded during the pull-out tests of untreated bamboo fibers are shown in Figure 6 as a function of $D_{f}$ (39 values, 12 regular plus 27 non-regular). Figure 6 provides 
an additional criterion for the initial selection of the fibers: they should not only be regularly-sized, defect-free and thick enough to resist the tensile stress applied to pull them out, but on the other side also thin enough to minimize the proportion of internal interfaces (connecting the elementary fibers and the fibrils to each other) with respect to the outer interface (connecting the fiber to the polymer). Thicker technical fibers have more extended outer surfaces, but also many more internal interfaces, which increases the probability of extraction by structural telescoping. The $F_{\max }$ values in Figure 6 are spread over a range of equivalent diameters from about $250 \mu \mathrm{m}$ to almost one millimeter, but regular tests were obtained only for samples processed with fibers having a diameter less than $500 \mu \mathrm{m}$, whatever the value of $L_{e m b}$. As a matter of fact, Da Costa et al. [41] already observed that, when performing tensile tests on bamboo fibers with the aim of Weibull analysis, thinner fibers showed a more uniform fracture associated with lesser fibrils, while fibers with larger diameters would display a relatively non-uniform fracture with participation of more fibrils. Statistically, the probability that one of the many fibrils of the thicker bamboo fiber would prematurely break and then act as a flaw to cause the fiber rupture at a lower stress is higher, when compared to the thinner fiber. As a consequence, it is more likely that larger diameter fibers have lower interfacial shear strength than the opposite.

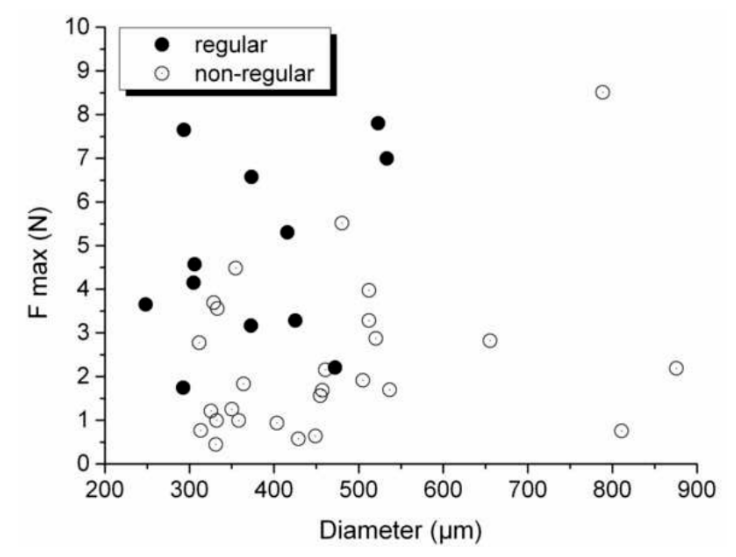

Figure 6. Values of maximum load $(F \max )(\mathrm{N})$ recorded by pull-out test on samples made of untreated bamboo fibers embedded in a PLA matrix. The values are plotted as a function of the fiber equivalent diameter $(\mu \mathrm{m})$ for both regular and non-regular samples.

Pull-out tests were also performed on mercerized bamboo fibers from the same batch. Figure $4 \mathrm{c}$ shows the surface of an untreated bamboo fiber. Mercerization modified the surface of the fibers both topographically and chemically. Figure $4 \mathrm{~d}$ shows that roughness changed and Figure $4 \mathrm{e}$ proves that the removal of the lignin loosened the bundles. The literature reports that $\mathrm{NaOH}$ solutions make the fibers swell by attacking and destroying their non-cellulosic structure [42]. Having the elementary fibers loosened is good when the objective is dispersing the bamboo fibers within a matrix to obtain the final green composite; however, in terms of pull-out tests, this condition is probably the most complicated to interpret. Unless the elementary fibers are completely isolated from each other, it is hard to quantify the extent of fiber surface actually interacting with the polymer and efficiently stressed during the pull-out test. In addition, Figure $4 \mathrm{f}$ shows that mercerization, if not followed by a thorough washing procedure, leaves residual salts on the surface of the fibers, which is a drawback of the treatment.

The chemical effects of mercerization were investigated by FTIR spectroscopy. Figure 7A shows the FTIR spectra of bamboo fibers between 2000 and $600 \mathrm{~cm}^{-1}$ : (a) before the treatment; (b) right after the treatment (without washing); and (c) when the treatment was followed by careful washing and filtering. Figure 7B reports the relative FTIR spectra between 4000 and $2000 \mathrm{~cm}^{-1}$, where only stretching vibration peaks of $=\mathrm{C}-\mathrm{H}$ and $-\mathrm{C}-\mathrm{H}$ are observed around 3300 and $2900 \mathrm{~cm}^{-1}$. 


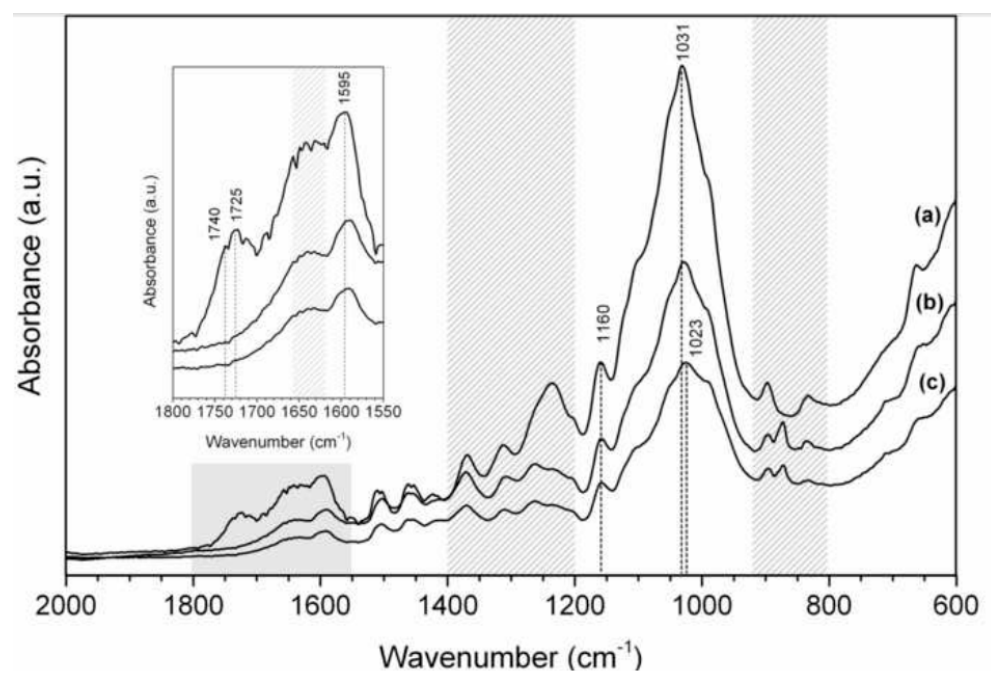

(A)

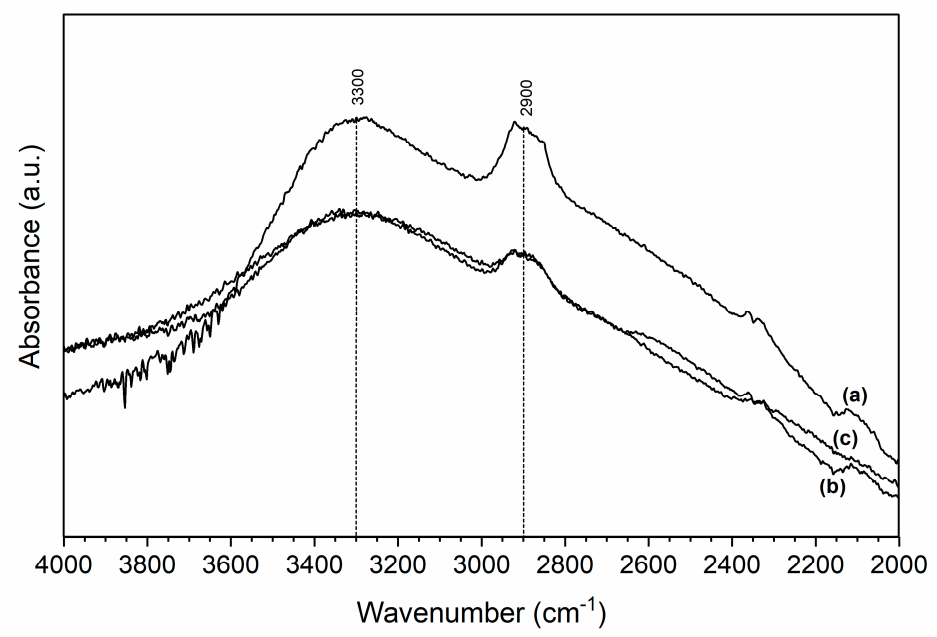

(B)

Figure 7. (A) FTIR spectra between 2000 and $600 \mathrm{~cm}^{-1}$ obtained for (a) bamboo fibers in their pristine state (untreated), (b) mercerized bamboo fibers (treated unwashed) and (c) mercerized bamboo fibers after washing plus filtering (treated washed filtered). The inset shows a zoom of the absorption bands from 1800 to $1550 \mathrm{~cm}^{-1}$. (B) FTIR spectra between 4000 and $2000 \mathrm{~cm}^{-1}$ obtained for (a) bamboo fibers in their pristine state (untreated); (b) mercerized bamboo fibers (treated unwashed) and (c) mercerized bamboo fibers after washing plus filtering (treated washed filtered).

The absorption bands were assigned on the basis of the data reported in the literature about different ligno-cellulosic materials (hemp hurds [43] and fibers [44], wheat straw nanofibers [45], oil palm fibers [46], bamboo fibers [47], wood [48,49]). In the range between $1800 \mathrm{~cm}^{-1}$ and $800 \mathrm{~cm}^{-1}$ it is possible to find evidence of the chemical modification of the fibers, mostly consisting of the removal of lignin and hemicellulose and the purification of crystalline cellulose. The inset zooms in a range showing a reduction of the bands typically assigned to hemicellulose $\left(1740 \mathrm{~cm}^{-1}\right)$ and lignin (1725 and $1595 \mathrm{~cm}^{-1}$, vibrations of the carbonyl groups and of the aromatic rings of phenyl propane, respectively). The hatched area on the inset identifies the absorption bands assigned to the bending vibration of the hydroxyl groups of water absorbed by holocellulose. The first hatched area on the main graph includes the stretching vibrations of the hydroxyl groups of water absorbed by holocellulose, but also the bending and stretching vibrations of $\mathrm{CH}$ and $\mathrm{CO}$ bonds in the aromatic rings of crystalline cellulose. The second hatched area contains the out-of-plane stretching vibrations of $\mathrm{CH}$ bonds in the 
aromatic rings of crystalline cellulose, as well as the stretching vibrations of $\mathrm{CH}$ and $\mathrm{COC}$ bonds in the $\beta-(1,4)$-glycosidic linkages between the anhydroglucose units. Polymorphism has already been documented for $\mathrm{NaOH}$-treated cellulose [50]. The absorption band at $1160 \mathrm{~cm}^{-1}$ is due to crystalline cellulose (COC asymmetrical stretching). The peak between $1150 \mathrm{~cm}^{-1}$ and $950 \mathrm{~cm}^{-1}$ contains many contributions: it is particularly noteworthy the slight shift of the main peak from $1031 \mathrm{~cm}^{-1}$ (in-plane deformation of $\mathrm{CH}$ bonds from lignin) to $1023 \mathrm{~cm}^{-1}$ (stretching vibration of $\mathrm{CO}$ bonds in crystalline cellulose), which could be a further sign of successful mercerization. Additionally, the smoothest FTIR spectra were recorded on the fibers that were carefully washed and filtered (Figure 7Ac), which is in agreement with the comment about Figure $4 \mathrm{f}$.

A total of 17 pull-out tests were performed on mercerized bamboo fibers; seven of them were considered as regular and interpreted, resulting in a value of $\tau_{a p p}=5.3 \pm 3.3 \mathrm{MPa}$ (lower than the value obtained for the untreated fibers). It appears that mercerization does not necessarily improve the interface between ligno-cellulosic fibers and polymers, even though previous literature suggests the opposite. An attempt to evaluate the influence of mercerization on the quality of the interface was made by interpreting the statistics of the two populations of data via the student's $t$-test. The test gave a value of $p=0.22$, meaning that in this case mercerization has no significant impact on the results (the limit being at 0.05 ). Scatter plots of the values of the apparent interfacial shear strength $\tau_{\text {app }}$ as a function of the equivalent diameter $D_{f}$ and the embedded length $L_{e m b}$ are provided in Figure 8 for both untreated and treated bamboo fibers. No obvious mathematical relationships can be pointed out. Figure $8 \mathrm{a}$ shows that, except for two points, the alkali treatment limits data dispersion by restraining the values of both the apparent diameters ( 350 to $400 \mu \mathrm{m}$ ) and the obtained apparent interfacial shear strength ( 3 to $5 \mathrm{MPa}$ ) to narrower ranges. As for the embedded length (Figure 8b), the alkali treatment had no statistical effect because $L_{e m b}$ depends only on sample processing, and the experimental procedure used in this work was designed with the purpose of controlling it. In conclusion, definite indications that mercerization is actually improving the interface between bamboo fibers and PLA matrix were not obtained in this work. The opposite result had been obtained on traditional oil-based polyester matrices despite the fact that the alkali treatment reduces the ductility of bamboo fibers [51]. It is proposed that the decreased ductility, together with the inherent brittleness of PLA, could have led to an early pull-out of the fibers, resulting in a weaker interface; but this would need to be confirmed in further studies.
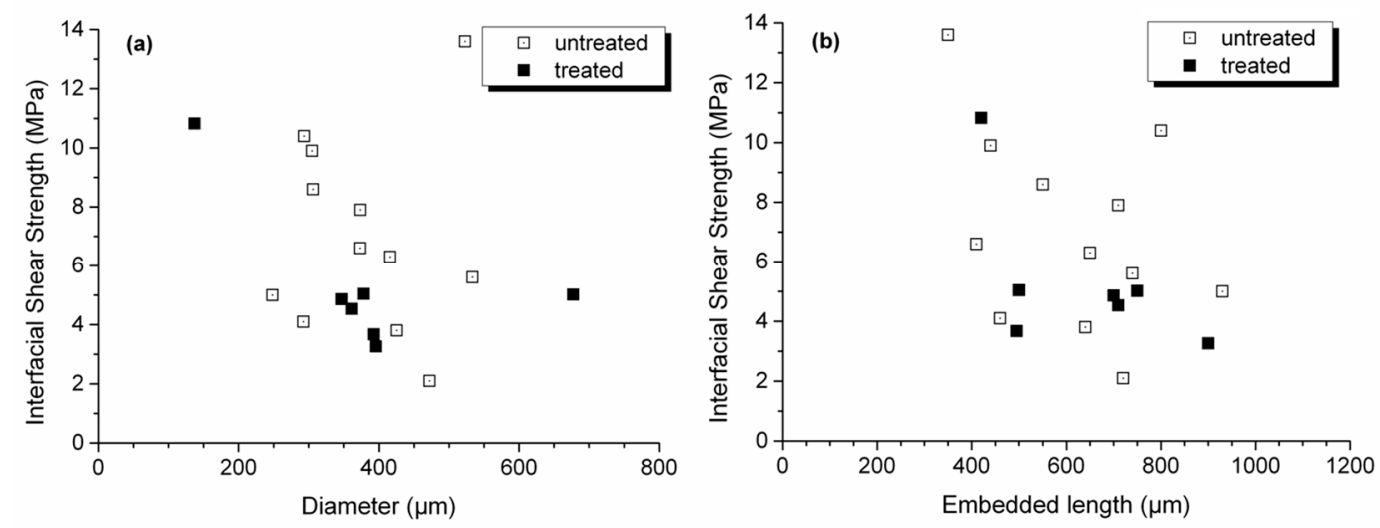

Figure 8. Apparent interfacial shear strength (MPa) between PLA and bamboo fibers (both untreated and mercerized) as a function of (a) equivalent diameter $(\mu \mathrm{m})$ and $(\mathbf{b})$ embedded length $(\mu \mathrm{m})$. Only regular samples are represented.

\section{Conclusions}

In this work, a procedure was described to measure the apparent interfacial shear strength from pull-out tests on both untreated and mercerized bamboo technical fibers from the same batch, 
embedded in the same poly(lactic acid) (PLA) matrix. It was found that to acquire sufficiently reliable measurements of interfacial shear strength on common batches of natural fibers in use in composites, an improved control on the length of fiber embedded in the polymer matrix is needed. The results of the pull-out tests were sorted and classified on the basis of several criteria: the shape of the load vs. displacement curves; the aspect of the fiber tips before and after the pull-out tests; and the hole left in the matrix by the pulled-out fiber. Data selection, treatment and interpretation were discussed by comparing the results obtained with reference to the occurrence or not of fibrillation during pull-out. The results showed that ideal bamboo fibers for pull-out tests should be not only regularly-sized (little or no variation of their cross-sectional shape and size as a function of the fiber length), defect-free on the outer surface, and thick enough to resist the tensile stress without breaking, but also thin enough to minimize the risk of extraction by structural telescoping and the occurrence of volume defects (fiber diameter should be in the range 250-500 $\mu \mathrm{m}$ ). Mercerization modified both the surface topography and chemical composition of the fibers, and eventually led to a finer control over the apparent fiber diameter, but did not improve the stress transfer at the interface with the polymer $\left(\tau_{\text {app }}=7.0 \pm 3.1 \mathrm{MPa}\right.$ and $5.3 \pm 2.4 \mathrm{MPa}$ for untreated and treated bamboo fibers respectively).

Acknowledgments: The authors would like to thank Benoît Lefez for acquiring FTIR spectra and Brahim Mazian for taking images of fiber cross-sections.

Author Contributions: Quentin Viel performed the experiments both in France and in the US, then processed the data. Antonella Esposito conceived and designed the experiments, then coordinated the discussion of the results within the group of collaborators. Jean-Marc Saiter obtained the raw fibers and made it possible for Quentin Viel to go to the US. Carlo Santulli contributed to the discussion of the results and the submission of the final manuscript. Joseph A. Turner provided the equipment and analysis tools that were used by Quentin Viel to perform the experiments, he also supervised the experiments and contributed to the discussion of the results. Quentin Viel and Antonella Esposito wrote the paper, which was successively proofread by all the co-authors. English proofreading was ensured by Joseph A. Turner.

Conflicts of Interest: The authors declare no conflict of interest.

\section{References}

1. Ji, S.G.; Cho, D.; Park, W.H.; Lee, B.C. Electron beam effect on the tensile properties and topology of jute fibers and the interfacial strength of jute-PLA green composites. Macromol. Res. 2010, 18, 919-922. [CrossRef]

2. Faruk, O.; Bledzki, A.; Fink, H.; Sain, M. Biocomposites reinforced with natural fibers. Prog. Polym. Sci. 2012, 37, 1552-1596. [CrossRef]

3. Andersons, J.; Sparnins, E.; Joffe, R. Stiffness and strength of flax fiber/polymer matrix composites. Polym. Compos. 2006, 27, 221-229. [CrossRef]

4. Saiter, J.M.; Esposito, A.; Dobircau, L.; Turner, J.A.; Adhikari, R. Synthetic polymer composites reinforced by bamboo fibers. Macromol. Symp. 2013, 327, 114-120. [CrossRef]

5. Hiziroglu, S.; Jarusombuti, S.; Bauchongkol, P.; Fueangvivat, V. Overlaying properties of fiberboard manufactured from bamboo and rice straw. Ind. Crop Prod. 2008, 28, 107-111. [CrossRef]

6. Abdul Khalil, H.P.S.; Bhat, I.; Jawaid, M.; Zaidon, A.; Hermawan, D.; Hadi, Y. Bamboo fibre reinforced biocomposites: A review. Mater. Des. 2012, 42, 353-368. [CrossRef]

7. Sato, J. People in between: Conversion and conservation of forest lands in Thailand. Dev. Chang. 2000, 31, 155-177. [CrossRef]

8. Sasaki, C.; Wanaka, M.; Takagi, H.; Tamura, S.; Asada, C.; Nakamura, Y. Evaluation of epoxy resins synthesized from steam-exploded bamboo lignin. Ind. Crop Prod. 2013, 43, 757-761. [CrossRef]

9. Lee, S.-H.; Wang, S. Biodegradable polymers/bamboo fiber biocomposite with bio-based coupling agent. Compos. Part A 2006, 37, 80-91. [CrossRef]

10. Takagi, H.; Ichihara, Y. Effect of fiber length on mechanical properties of "green" composites using a starch-based resin and short bamboo fibers. JSME Int. J. 2004, 47, 551-555. [CrossRef]

11. Tung, N.H.; Yamamoto, H.; Matsuoka, T.; Fujii, T. Effect of surface treatment on interfacial strength between bamboo fiber and PP resin. JSME Int. J. 2004, 47, 561-565. [CrossRef]

12. Trujillo, E.; Moesen, M.; Osorio, L.; Van Vuure, A.W.; Ivens, J.; Verpoest, I. Bamboo fibres for reinforcement in composite materials: Strength Weibull analysis. Compos. Part A 2014, 61, 115-125. [CrossRef] 
13. Huang, Y.; Fei, B.; Wei, P.; Zhao, C. Mechanical properties of bamboo fiber cell walls during the culm development by nanoindentation. Ind. Crop Prod. 2016, 92, 102-108. [CrossRef]

14. Garlotta, D. A literature review of poly(lactic acid). J. Polym. Environ. 2001, 9, 63-84. [CrossRef]

15. Di Francia, C.; Ward, T.; Claus, R. The single-fibre pull-out test. Review and interpretation. Compos. Part $A$ 1996, 27, 597-612. [CrossRef]

16. Stamboulis, A.; Baillie, C.; Schulz, E. Interfacial characterisation of flax fibre-thermoplastic polymer composites by the pull-out test. Angew. Makromol. Chem. 1999, 272, 117-120. [CrossRef]

17. Graupner, N.; Rößler, J.; Ziegmann, G.; Müssig, J. Fibre/matrix adhesion of cellulose fibres in PLA, PP and MAPP: A critical review of pull-out test, microbond test and single fibre fragmentation test results. Compos. Part A 2014, 63, 133-148. [CrossRef]

18. Sydenstricker, T.H.D.; Mochnaz, S.; Amico, S.C. Pull-out and other evaluations in sisal-reinforced polyester biocomposites. Polym. Test. 2003, 22, 375-380. [CrossRef]

19. Chen, H.; Miao, M.; Ding, X. Influence of moisture absorption on the interfacial strength of bamboo/vinyl. Compos. Part A 2009, 40, 2013-2019. [CrossRef]

20. Tokoro, R.; Vu, D.; Okubo, K.; Tanaka, T.; Fujii, T.; Fujiura, T. How to improve mechanical properties of polylactic acid with bamboo fibers. J. Mater. Sci. 2008, 43, 775-787. [CrossRef]

21. Fuentes, C.A.; Brughmans, G.; Tran, L.Q.N.; Dupont-Gillain, C.; Verpoest, I.; Van Vuure, A.W. Mechanical behaviour and practical adhesion at a bamboo composite interface: Physical adhesion and mechanical interlocking. Compos. Sci. Technol. 2015, 109, 40-47. [CrossRef]

22. Pisanova, E.; Zhandarov, S.; Mäder, E. How can adhesion be determined from micromechanical tests? Compos. Part A 2001, 32, 425-434. [CrossRef]

23. Zhandarov, S.; Pisanova, E.; Mäder, E.; Nairn, J.A. Investigation of load transfer between the fiber and the matrix in pull-out tests with fibers having different diameters. J. Adhes. Sci. Technol. 2001, 15, 205-222. [CrossRef]

24. Zixuan, D.R.; Xuexi, Y.; Hankun, Z.; Hao, W.; Yu, W.Y. Quantitative characterization of the interface between bamboo fiber and polypropylene with pull-out test and nanomechanical imaging. J. Mater. Sci. 2017, 52, 1296-1307.

25. Ramyarangsi, S. Bamboo research in Thailand. In Proceedings of the International Bamboo Workshop, Hangzhou, China, 6-14 October 1985.

26. Sharma, Y.M.L. Inventory and Resources of Bamboos. In Proceedings of the International Bamboo Workshop, Hangzhou, China, 6-14 October 1985.

27. Maxwell, J.F.; Elliott, S.; Palee, P.; Anysarnsunthorn, V. The vegetation of Doi Khuntan National Park, Lamphun-Lampang Provinces, Thailand. Nat. Hist. Bull. Siam Soc. 1995, 43, 185-205.

28. Maxwell, J.F. A Synopsis of the Vegetation of Thailand. Nat. Hist. J. Chulalongkorn Univ. 2004, 4, 19-29.

29. Prachayo, B. Farmers and Forests: A Changing Phase in Northeast Thailand. Southeast Asian Stud. 2000, 138, 271-446.

30. Marod, D.; Kutintara, U.; Yarwudhi, C.; Tanaka, H.; Nakashisuka, T. Structural dynamics of a natural mixed deciduous forest in western Thailand. J. Veg. Sci. 1999, 10, 777-786. [CrossRef]

31. Larpkern, P.; Moe, S.R.; Totland, Ø. The effects of environmental variables and human disturbance on woody species richness and diversity in a bamboo-deciduous forest in northeastern Thailand. Ecol. Res. 2009, 24, 147-156. [CrossRef]

32. Osorio, L.; Trujillo, E.; Van Vuure, A.W.; Verpoest, I. Morphological aspects and mechanical properties of single bamboo fibers and flexural characterization of bamboo/epoxy composites. J. Reinf. Plast. Compos. 2011, 30, 396-408. [CrossRef]

33. Liu, Y.; Hu, H. X-ray Diffraction Study of Bamboo Fibers Treated with NaOH. Fibers Polym. 2008, 9, 735-739. [CrossRef]

34. Greszczuk, L. Theoretical studies of the mechanics of the fiber-matrix interface in composites. In Interfaces in Composites; American Society for Testing and Materials: West Conshohocken, PA, USA, 1969; p. 42.

35. Rao, K.M.M.; Rao, K.M. Extraction and tensile properties of natural fibers: Vakka, date and bamboo. Compos. Struct. 2007, 77, 288-295. [CrossRef]

36. Martin, N.; Mouret, N.; Davies, P.; Baley, C. Influence of the degree of retting of flax fibers on the tensile properties of single fibers and short fiber/polypropylene composites. Ind. Crop Prod. 2013, 49, 755-767. [CrossRef] 
37. Fuentes, C.; Tran, L.; Van Hellemont, M.; Janssens, V.; Dupont-Gillain, C. Effect of physical adhesion on mechanical behaviour of bamboo fibre reinforced thermoplastic composites. Colloids Surf. A Physicochem. Eng. Asp. 2013, 418, 7-15. [CrossRef]

38. Tanaka, C.; Okubo, K.; Fujii, T. Effective Degree of Fibrillation of Micro-Fibrillated Bamboo Fiber Processed by Stone Mill for Improving Mechanical Properties of PLA Composite. JSMS J. Soc. Mater. Sci. Jpn. 2009, 58, 368-373. [CrossRef]

39. Le Duigou, A.; Davies, P.; Baley, C. Interfacial bonding of flax fibre/poly(L-lactide) bio-composites. Compos. Sci. Technol. 2010, 70, 231-239. [CrossRef]

40. Wong, S.; Shanks, R.; Hodzic, A. Effect of additives on the interfacial stregth of poly(L-lactic acid) and poly(3-hydroxy butyric acid)-flax fibre composites. Compos. Sci. Technol. 2007, 67, 2478-2484. [CrossRef]

41. Da Costa, L.L.; Loiola, R.L.; Monteiro, S.N. Diameter dependence of tensile strength by Weibull analysis: Part I bamboo fiber. Matéria 2010, 15, 110-116. [CrossRef]

42. Das, M.; Chakraborty, D. Influence of alkali treatment on the fine structure and morphology of bamboo fibers. J. Appl. Polym. Sci. 2006, 102, 5050-5056. [CrossRef]

43. Gandolfi, S.; Ottolina, G.; Riva, S.; Pedrocchi Fantoni, G.; Patel, I. Complete chemical analysis of Carmagnola hemp hurds and structural features of its components. Bioresources 2013, 8, 2641-2656. [CrossRef]

44. Kabir, M.M.; Wang, H.; Lau, K.T.; Cardona, F. Effects of chemical treatments on hemp fibre structure. Appl. Surf. Sci. 2013, 276, 13-23. [CrossRef]

45. Alemdar, A.; Sain, M. Biocomposites from wheat straw nanofibres: Morphology, thermal and mechanical properties. Compos. Sci. Technol. 2008, 68, 557-565. [CrossRef]

46. Sreekala, M.; Kumaran, M.; Joseph, S.; Jacob, M. Oil palm fibre reinforced phenol formaldehyde. Appl. Compos. Mater. 2000, 7, 235-329. [CrossRef]

47. Wen, J.; Xue, B.; Suna, R.; Pinkert, A. Unmasking the structural features and property of lignin from bamboo. Ind. Crop Prod. 2012, 42, 332-343. [CrossRef]

48. Chen, H.; Ferrari, C.; Angiuli, M.; Yao, J.; Raspi, C.; Bramanti, E. Qualitative and quantitative analysis of wood samples by Fourier transform infrared spectroscopy and multivariate analysis. Carbohydr. Polym. 2010, 82, 772-778. [CrossRef]

49. Corgié, S.C.; Smith, H.M.; Walker, L.P. Enzymatic transformations of cellulose assessed by quantitative high-throughput Fourier transform infrared spectroscopy (QHT-FTIR). Biotechnol. Bioeng. 2011, 108, 1509-1520. [CrossRef] [PubMed]

50. Gupta, P.K.; Uniyal, V.; Naithani, S. Polymorphic transformation of cellulose I to cellulose II by alkali pretreatment and urea as an additive. Carbohydr. Polym. 2013, 94, 843-849. [CrossRef] [PubMed]

51. Wong, K.J.; Yousif, B.F.; Low, K.O. The effects of alkali treatment on the interfacial adhesion of bamboo fibres. J. Mater. Des. Appl. 2010, 224, 139-148. [CrossRef] 